\title{
Characterization of Liquid Suspensions in 3D using Environmental Scanning Electron Microscopy in Transmission
}

\author{
Juan Xiao ${ }^{1}$, Lucian Roiban ${ }^{1}$, Geneviève Foray ${ }^{1}$, and Karine Masenelli-Varlot ${ }^{1}$ \\ 1. Univ Lyon, INSA-Lyon, Université Claude Bernard Lyon 1, MATEIS, CNRS UMR 5510, 69621 \\ Villeurbanne, France.
}

Liquid-phase electron microscopy has been pushed forward during the last decade thanks to two major developments. On the one hand, liquids can be encapsulated into closed cells and analyzed in electron microscopes operating under vacuum. When compared with cryo-electron microscopy techniques, such experiments have allowed the study of nanoparticle dynamics such as in situ motion, crystallization of evolution during electrochemical processes [1]. On the other hand, the sample temperature and gas pressure can be tuned in environmental electron microscopes so as to preserve the liquid state by staying on the dew curve [2]. Interestingly, observing a liquid suspension in transmission in an Environmental Scanning Electron Microscope (STEM-in-ESEM mode) gives access to the nanoparticles dispersed in the liquid with a resolution down to a few nanometers [3]. This imaging mode is very interesting when compared with sealed cells, as it is possible to monitor structural changes upon dehydration. It is also possible to tilt the sample and get information in three dimensions [4].

We will focus on an aqueous suspension containing latex particles (copolymer derived from styrene and metacrylic acid esters) of diameter around $200 \mathrm{~nm}$. This type of material is peculiar as spheres can only be observed in the native liquid state: upon drying, the spheres are deformed and form a uniform film (non-reversible process). We will show image series acquired in the STEM-in-SEM mode, using an annular dark field detector, for different orientations of the liquid sample. They will be analyzed in terms of contrast, by comparison with Monte Carlo simulation results, and resolution [5]. The absence of particle motion and the stability of the water film (including irradiation damage effects) will also be discussed.

We will present reconstructed volumes (see Figure 1) and 3D models (see Figure 2) showing the latex spheres and the associated surfactant distribution. Several methods will be compared for the measurement of the spatial resolution. The morphological parameters, such as the lattice type, grain sizes, or distance to first neighbours, will be quantified in 3D and compared with results obtained with cryo-SEM [6].

\section{References:}

[1] F. Ross Ed. Liquid cell electron microscopy (advances in microscopy and microanalysis) (Cambridge University Press, UK) 2016.

[2] D. Stokes. Principles and practice of variable pressure: environmental scanning electron microscopy (VP-ESEM) (Wiley-Blackwell, RMS) 2008.

[3] A. Bogner et al., Ultramicroscopy 104 (2005) p. 290.

[4] P. Jornsanoh et al., Ultramicroscopy 111 (2011) p. 1247.

[5] J. Xiao, G. Foray, K. Masenelli-Varlot. J. Microscopy 269 (2018) p. 151.

[6] The authors acknowledge the Consortium Lyon Saint-Etienne de Microscopie (CLYM) for the access to the microscope, the China Scholarship Council (CSC) and Institut Universitaire de France (IUF) for financial support, and BASF for having provided the samples. 

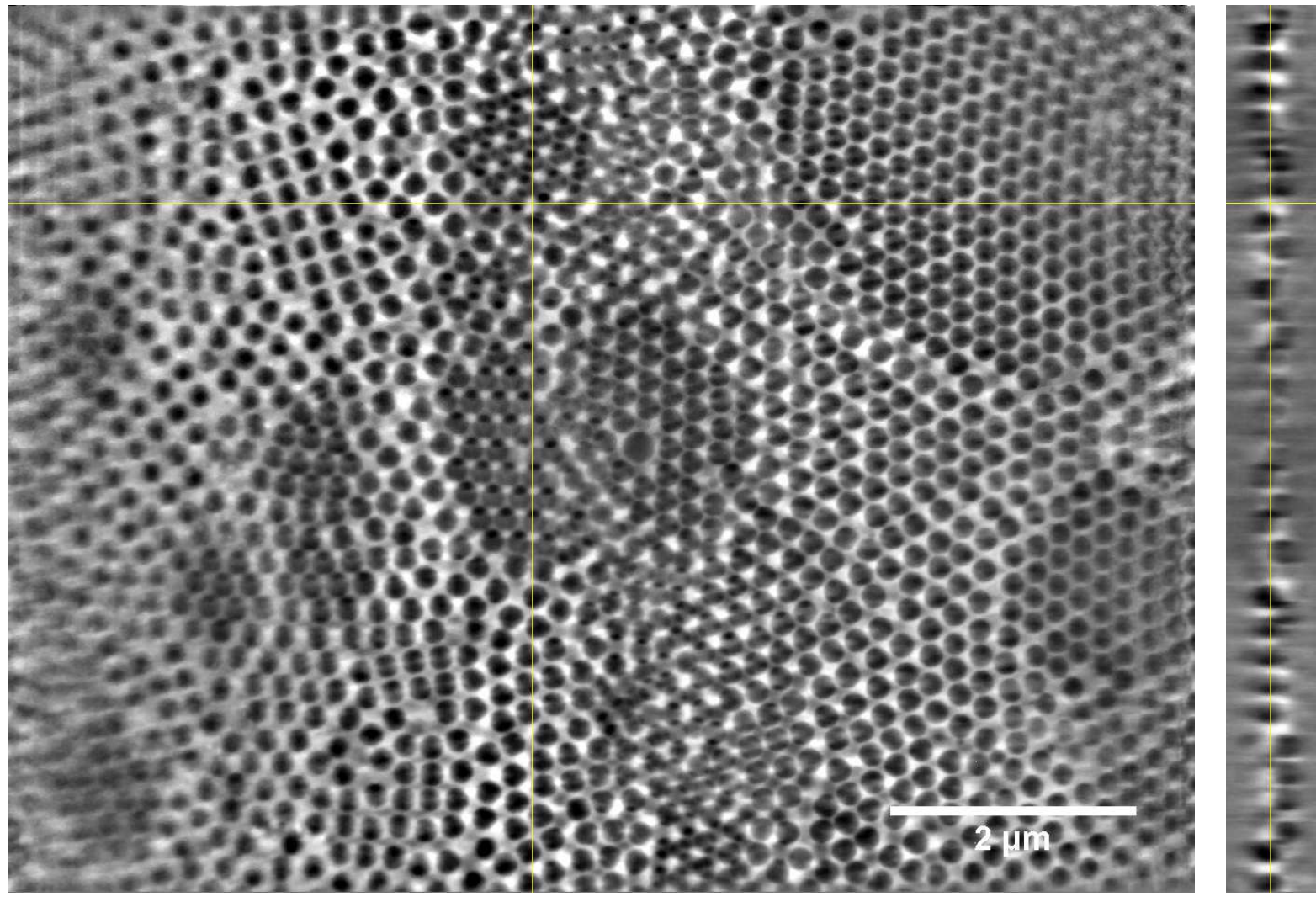

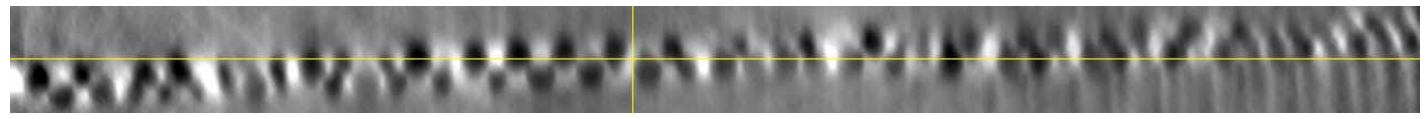

Figure 1. Orthogonal cross sections through the reconstructed volume. The latex particles (in black) arrange themselves in two layers on the holey carbon membrane.
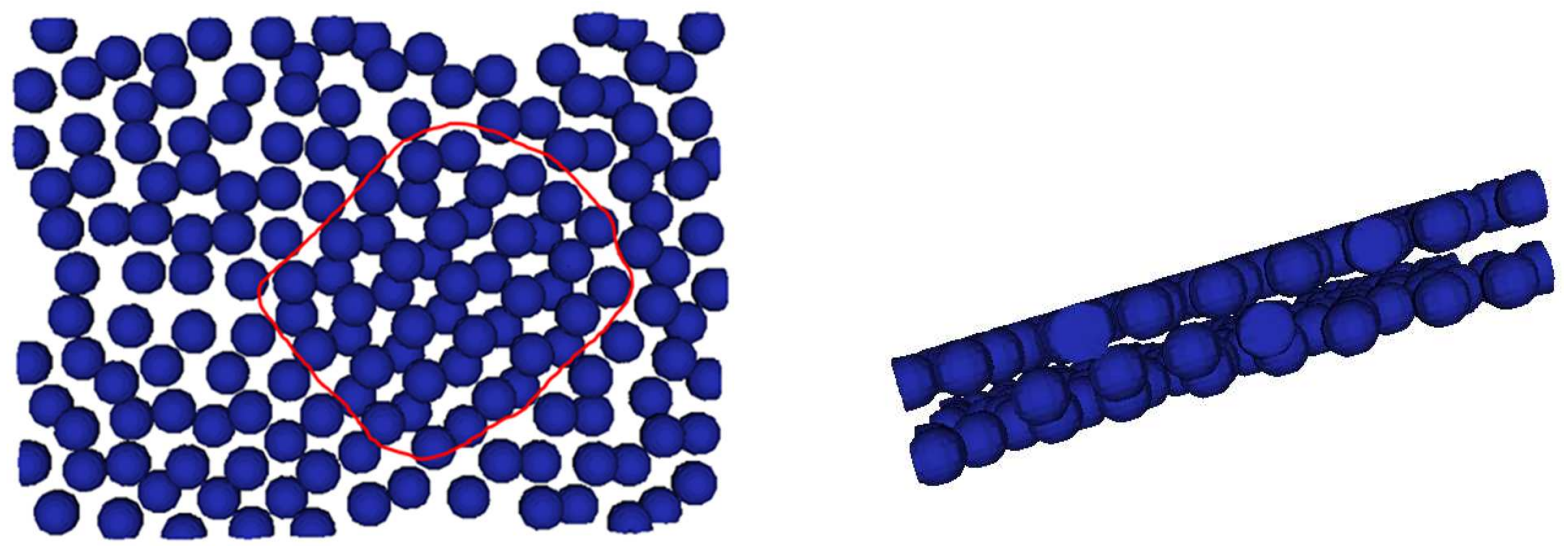

Figure 2. Top and side views of the reconstructed volume obtained after segmentation. The position of a hole on the supporting carbon membrane is indicated. 